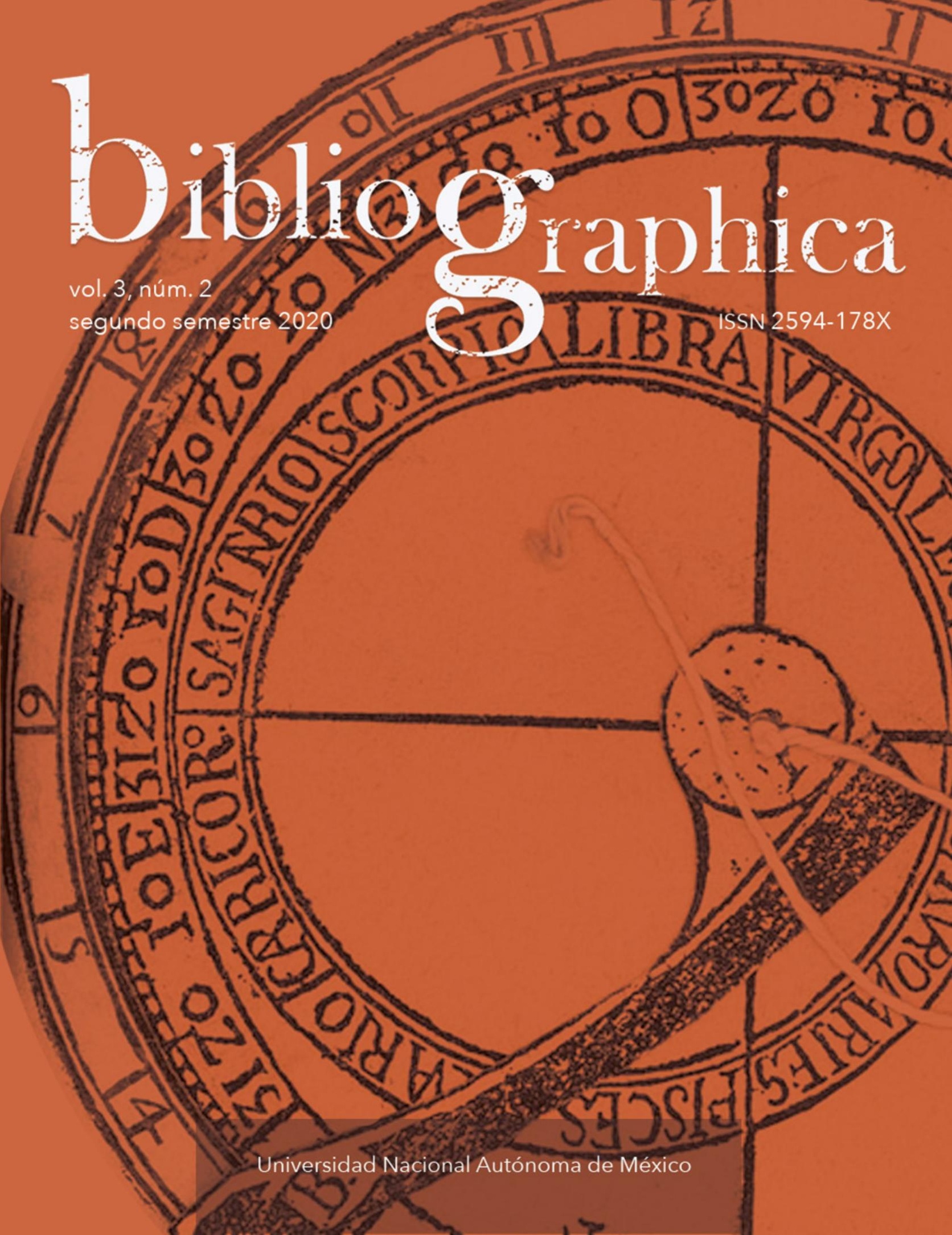




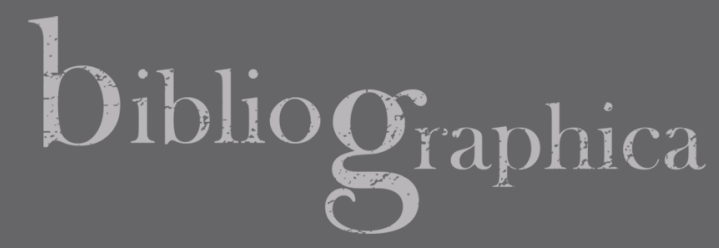

\section{Advertencia editorial}

\section{Manuel Suárez Rivera}

manuelsr@unam.mx

\section{Laurette Godinas}

lgodinas@unam.mx

Universidad Nacional Autónoma de México

Instituto de Investigaciones Bibliográficas

DOI: https://doi.org/10.22201/iib.2594178xe.2020.2.88 
Para su sexto número, y tal vez buscando inconscientemente el apoyo de las estrellas para combatir la adversidad de este 2020 -que en el zodiaco chino, como año de la Rata de Metal, auguraba cambios vertiginosos en todos los ámbitos-, hemos escogido para la identidad gráfica de nuestra revista una obra que pertenece a la literatura de divulgación cronológica y astrológica: el Reportorio de los tiempos e historia natural de esta Nueva España, de Enrico Martínez. ${ }^{1}$ La Biblioteca Nacional de México posee esta obra, la cual se localiza en la Sala Mexicana con la clasificación RSM 1606 M4MAR. El ejemplar, encuadernado en pergamino y sin marcas evidentes de procedencia, presenta ahora, después de la restauración de su portada, un estado excelente de conservación y puede ser consultado en formato digital en la Biblioteca Nacional Digital de México, que inauguró a principios de julio de este año su nueva interfaz.

El Reportorio de los tiempos, que incluye el grabado sobre el cual volveremos un poco más adelante, consta de cinco tratados que dan cuenta del interés de su autor -oriundo del puerto de Hamburgo y originalmente llamado Heinrich Martin-2 por el estudio de los fenómenos físicos, una inquietud que le valió el nombramiento de cosmógrafo real. ${ }^{3}$ El primer tratado ofrece una descripción de los cuerpos celestes y el planteamiento de varias cuestiones astrológicas. El segundo propone un panorama general del desarrollo histórico de Nueva España. En el tercero, Enrico Martínez da libre curso a su afán de "ilustrar al vulgo en todos aquellos asuntos físicos y morales dignos de interés", ${ }^{4}$ orientándolo hacia las particularidades astrológicas, geográficas y físicas novohispanas. El cuarto da cuenta de la importancia de la astrología aplicada a los cuidados medicinales, mientras que el quinto trata específicamente de la conjunción de Júpiter y Saturno, donde explica el fenómeno de un modo ligeramente distinto

\footnotetext{
${ }^{1}$ Enrico Martínez, Reportorio de los tiempos, y historia natvral desta Nveva España. Compuesto por Henrrico Martinez Cosmographo de su Magestad è Interprete del Sancto Officio deste Reyno. Dirigido al Excelentissimo Señor Don luan de Mendoça y Luna Marques de Montesclaros, Virrey, Gouernador, Presidente y Cappitan General por el Rey nuestro Señor en esta Nueua España (México: Imprenta de Enrico Martínez, 1606).

2 Según afirmaciones de Wilhelm Pferdekamp, Deutsche im frühen Mexiko (Stuttgar; Berlin: Verlags-Anstalt, 1938) y Francisco de la Maza, Enrico Martínez, cosmógrafo e impresor de Nueva España (México: Sociedad Mexicana de Geografía y Estadística, 1943).

3 José Enrique Covarrubias, "Henrico Martínez", en Rosa Camelo y Patricia Escandón, coords., Historiografía mexicana. Volumen II. La creación de una imagen propia. La tradición española. Tomo 1: Historiografía civil (México: UNAM, 2012), 395.

${ }^{4}$ Ibid., 396.
} 
a lo propuesto por Kepler, y sigue al ilustre profesor salmantino Pedro Ciruelo. ${ }^{5}$ Enrico Martínez agrega al final una sección titulada "Breve relación del tiempo en el que han sucedido algunas cosas notables y dignas de memoria así en la Nueva España como en los reinos de Castilla y otras partes del mundo desde el año 1520 y hasta 1590", sacadas, de acuerdo con el propio autor, "de las crónicas y de historias de autores fidedignos".

Sin duda, la obra es muy completa en cuanto a cuestiones astrológicas y permite conocer vívidamente el paradigma aristotélico que predominó en este tipo de publicaciones durante varios siglos hasta bien entrado el XIX. Como sus modelos, Jerónimo de Chaves con su Chronographia o reportorio de los tiempos, el más copioso y preciso, que hasta a hora ha salido a luz, publicada por primera vez en 1584 y con por lo menos 3 ediciones posteriores, y Rodrigo Zamorano con su Cronología y reportorio de la razón de los tiempos, con edición única en 1594, ambas en Sevilla, Enrico Martínez da cuenta a lo largo de su tratado de la lógica que imperaba en el ámbito médico-astrológico. Así, explica en su capítulo VIII del tratado cuarto que:

Bien sabido es que los humores de nuestros cuerpos tienen correspondencia con las calidades de los cuatro elementos, y que también los cuerpos celestes influyen las calidades de los elementos en todas las cosas corpóreas de este mundo, las cuales según Aristóteles están subordenadas a la región celeste, de donde se sigue, que así por el movimiento del sol y los demás planetas por el zodiaco, y según y que por sus conjunciones y aspectos se alteran los elementos y se aumentan a veces las calidades de alguno de ellos, causando destemplancia por calor, frío, humedad o sequedad, casi de la misma manera en su proporción se alteran también los humores de nuestros cuerpos, por la semejanza que tienen con las referidas calidades de los elementos y por lo consiguiente con la influencia celeste. ${ }^{6}$

\footnotetext{
${ }^{5}$ Como se encuentra detallado en el artículo de Marco Antonio Moreno Corral, "La astronomía en México en el siglo XVII", las enseñanzas del ilustre profesor salmantino le permiten estudiar el fenómeno de la conjunción de Júpiter y Saturno a la luz de otra conjunción planetaria, con una visión mucho más ortodoxa. Revista Ciencias 54 (abril-junio de 1999): 53. ${ }^{6}$ Martínez, Reportorio de los tiempos, 195. Los criterios para las citas incluyen la modernización de la puntuación y acentuación, y la uniformación de la vacilación gráfica entre $\mathrm{i} / \mathrm{j} / \mathrm{y}$ y $u / v / b$, así como la modernización ortográfica de los grupos consonánticos cultos y del uso de la h; se resuelven también -según el uso moderno- las contracciones entre preposición y determinante o pronombre y el uso de las mayúsculas.
} 
Sin embargo, como bien lo subraya Martha Tappan, si las obras de Chaves y Zamorano se desarrollan "conforme a los horizontes de expectativa de los géneros abordados", viendo al público de los repertorios como muy distinto al de sus obras de astronomía y circunscribiéndose "a las esferas de interés de sus lectores", proveyéndolos de información útil, Martínez opta por dar en su Reportorio de los tiempos datos de índole mucho más "curiosa", 7 como lo evidencia su interés por vincular la astronomía con la historia.

Otro interesante aspecto, material esta vez, que distingue la obra de otros repertorios similares es que Martínez incluyó en el capítulo XXVI del segundo tratado, titulado "En que se enseña cómo podrá una persona saber en qué signo nació", un instrumento para calcular el signo zodiacal bajo el cual nació una persona, en una lámina que nos inspiró para la identidad gráfica del presente número de Bibliographica.

Con una lógica implacable, afirma que sería de "poco fruto" haber dado a conocer las propiedades e inclinaciones de cada signo sin proporcionar una regla por dónde saber en qué signo nació cada quien; cabe destacar que se refiere no al signo zodiacal solar, relativamente fácil de conocer, sino lo que conocemos hoy como el ascendente porque, para Martínez: "decir ser una persona nacida en tal o tal signo, no es otra cosa que decir que al tiempo que la tal persona nació subía por el horizonte del oriente tal signo". ${ }^{8}$ Asimismo, el autor declara que, con el propósito de conocer el signo zodiacal, insertó en su obra un "instrumento, que es el modo más fácil que para ello he podido hallar", y acompaña esta afirmación con una sencilla explicación que da cuenta del funcionamiento de tal herramienta. Inicia por la presentación de sus partes:

El horizonte del oriente en este instrumento es la raya que está a modo de arco desde la letra A (que está en el centro del instrumento) hasta la letra B.

El espacio incluido entre los dos círculos exteriores se llama el círculo de las horas, el cual está repartido en 24 partes iguales por las 24 horas del día natural, y comienza la cuenta desde la mano izquierda hacia la derecha; y las horas de la parte de arriba son horas del día y las de la parte de o [sic] abajo son horas de noche, conforme lo enseña el letrero que va puesto al derredor del dicho círculo.

\footnotetext{
${ }_{7}^{7}$ Martha Tappan Velázquez, "Representaciones de la Tierra en un género de escritura del siglo XVI", Fuentes Humanísticas 26, núm. 47 (segundo semestre de 2013): 23.

${ }^{8}$ Martínez, Reportorio de los tiempos, 35-36.
} 
La parte exterior de la rueda de dentro, que se mueve a la redonda, está repartida en doce partes que denotan los doce meses del año y cada mes está repartido en quince partes de suerte que cada parte o rayazuela denota dos días.

Las otras doce divisiones o repartimentos de más adentro puestos en esta rueda movible representan los doce signos del zodiaco y en cada una de ellas se verá escrito el nombre de tal signo.

Después, Martínez da a conocer las instrucciones para su uso:

Entendido las partes de este instrumento y queriendo por él una persona saber en qué signo nació, ha de saber primeramente el mes, día y hora de su nacimiento, lo cual sabido buscará la tal hora en el círculo de las horas, y sobre ella pondrá el hilo que está asido en el centro del instrumento, de suerte que esté tirante, y teniéndolo así, moverá la rueda hasta tanto que el mes y día de su natividad esté debajo del hilo y estando la rueda de esta manera, verá qué signo viene a estar en la raya que va de $A$ a la $B$, que se dijo representar el horizonte, y ese tal se dirá ser el signo en que nació. ${ }^{9}$

Esta obra de Enrico Martínez representó un momento significativo en la historia de la pronosticación y de la práctica astrológica novohispana, que contó con grandes cosmógrafos como Carlos de Sigüenza y Góngora, ${ }^{10}$ fray Isidoro Alfonso de Castaneira, ${ }^{11}$ Pedro de Alarcón ${ }^{12}$ y, más tarde, Felipe de Zúñiga y Ontiveros, ${ }^{13}$ entre muchos otros. Como tal, y debido al valioso y único instrumento que incluyó, se trata de una obra singular y de gran valor.

\footnotetext{
${ }^{9}$ lbid., 35-37.

10 Para un análisis de Sigüenza, en tanto autor de almanaques, ver Margarita PerazaRugeley, "Llámenme 'el mexicano': los almanaques y otras obras de Carlos de Sigüenza y Góngora", en Currents in comparative Romance Languages and Literatures (Nueva York: Peter Lang, 2013), 215.

${ }^{11}$ Castaneira publicó ininterrumpidamente sus calendarios entre 1689 y 1720 . El título solía ser genéricamente: Kalendarium Franciscanum, ad formam, pro nobis antiquitus editam. ${ }^{12}$ Además de preparar sus Ephemeris y Calendarios, fue el titular de la cátedra de astrología y matemáticas en la Real Universidad desde junio de 1737 hasta 1752, cuando lo sustituyó Antonio Gamboa y Riaño. El primer almanaque conocido de Alarcón data del año 1724 y se tituló Nueva efemeris anunciada para los temporales de 1724, mientras que el último fue publicado bajo el título de Ephemeris del año del señor 1748. Bissexto: según el meridiano de México, por el Dr. D. Pedro Alarcon, cathedratico de mathematicas en esta pontificia, y real Universidad; es decir, Alarcón los publicó por espacio de 24 años.

${ }^{13}$ La labor de Felipe de Zúñiga como calendarista -la cual heredó a su hijo Marianoes muy significativa, pues tuvo el privilegio de hacerla con exclusividad durante las últimas
} 
Sin duda, dicho valor se ve aun acrecentado por el carácter polifacético de su autor y su participación en varios rubros de la vida cultural de Nueva España: en efecto, además de ser cosmógrafo, Enrico Martínez fue también arquitecto -se le recuerda en la Ciudad de México como el maestro mayor de las obras del desagüe- ${ }^{14} y$, sobre todo, impresor. A pesar de que llegó a esta última profesión por azar, tras recibir como segundo depositario la imprenta del holandés Adriano Cornelio César, que había sido confiscada por el Santo Oficio, Martínez logró dominar el arte de la imprenta y dio pie a lo que Francisco de la Maza considera una "copiosa obra de divulgación religiosa, como novenas, jaculatorias y oraciones".15

Esta trayectoria como empresario editorial e impresor duró 12 años (de 1599 a 1611) y se caracterizó sobre todo "por la destreza en el uso de la tipografía, que posibilitó que sus impresos, aunque sencillos, conserven una acertada y cuidada composición". ${ }^{16} \mathrm{Al}$ respecto, sin duda alguna el Reportorio de los tiempos es, con creces, la obra más original y ambiciosa del autor, que fue también su impresor.

Llama la atención que este impreso presente, en comparación con el resto de la producción de Enrico Martínez, una factura tipográfica primitiva, caracterizada por numerosas manchas de tinta o encabalgamientos de tipos. Aun así, tener uno de estos valiosos tesoros bibliográficos y ponerlo a disposición del público es un honor para la Biblioteca Nacional de México; que luzca como identidad gráfica de este número - gracias a la destacada labor creativa de Carolina Silva Bretón (banner) e Hilda Maldonado (portada) - representa un justo tributo a su relevancia en la historia de la cultura escrita en México. bg

cinco décadas del régimen virreinal. En general, publicó tres tipos de obras: las Efemérides calculadas y pronosticadas según el Meridiano de México, el Calendario manual y la Guía de Forasteros. En ocasiones publicaba las dos últimas en conjunto; por ejemplo, el Calendario manual y Guía de forasteros para el año de 1762. Para un estudio más profundo sobre los Zúñiga como autores de almanaques, ver Manuel Suárez Rivera, Dinastía de tinta y papel. Los Zúñiga Ontiveros en la cultura novohispana (1756-1825) (México: UNAM, IIB, 2019).

${ }^{14}$ Existen trabajos específicos sobre la vida de Enrico Martínez, entre los cuales destaca sin duda el de Francisco de la Maza, Enrico Martínez. Cosmógrafo e impresor de la Nueva España (México: Sociedad Mexicana de Geografía e Historia, 1943), reeditado en 1991 por el Instituto de Investigaciones Bibliográficas; para una presentación breve de su participación en la obra de desagüe, véase Jorge Vázquez Ángeles, "Enrique Martínez y Adrian Boot, ingenieros del fracaso", Casa del Tiempo, núm. 65 (marzo de 2013): 49-51.

${ }^{15}$ Francisco de la Maza, Enrico Martínez, 169.

${ }^{16}$ Nuria Lorente, "La imprenta en México. Enrico Martínez: impresor, intérprete y cosmógrafo del rey", Cuadernos del Hipogrifo. Revista de Literatura Hispanoamericana y Comparada 12 (2019): 68. 